\title{
Avifauna de la selva baja caducifolia en la cañada del río Sabino, Oaxaca, México
}

\author{
Avifauna of the tropical dry forest in the Sabino Canyon, Oaxaca, México
}

\author{
Leopoldo Vázquez ${ }^{1,3}$, Héctor Moya ${ }^{2}$ y María del Coro Arizmendi ${ }^{*}$ \\ ${ }^{1}$ Laboratorio de Ecología, Unidad de Biotecnología y Prototipos, Facultad de Estudios Superiores Iztacala, Universidad Nacional Autónoma de \\ México. Avenida de los Barrios \# 1, Los Reyes Ixtacala, 05490, Tlalnepantla, Estado de México, México. \\ ${ }^{2}$ Departamento de Ecología de Comunidades, Dirección de Investigación de Ordenamiento Ecológico y Conservación de los Ecosistemas, Instituto \\ Nacional de Ecología, Secretaría de Medio Ambiente y Recursos Naturales. Periférico 5000, Col. Insurgentes Cuicuilco, Coyoacán, 04530, México \\ D.F. \\ ${ }^{3}$ Dirección actual: Instituto de Biología, Universidad Nacional Autónoma de México. Apartado postal 70-233, 04510 México, D.F., México. \\ *Correspondencia: coro@servidor.unam.mx
}

\begin{abstract}
Resumen. Se describe la avifauna de la selva baja caducifolia del cañón del río Sabino, Oaxaca, al sureste de la Reserva de la Biosfera Tehuacán-Cuicatlán y compara la similitud de la avifauna de la Reserva con la de otras regiones con selvas bajas en México. El trabajo se realizó entre junio de 2005 y octubre de 2006, registrando 113 especies pertenecientes a 13 órdenes y 34 familias; 6 especies son muy abundantes, 10 abundantes, 20 comunes, 32 poco comunes y 37 raras; 79 de las especies registradas fueron residentes, 28 visitantes invernales, 2 visitantes de verano, 3 transitorias y 5 residentes con movimientos estacionales; 15 especies son endémicas a México y 23 están dentro de alguna categoría de riesgo. La avifauna estudiada representa una mezcla de especies características de la cuenca del Balsas, las vertientes pacífica y atlántica y los ambientes montanos y áridos del centro de México. Por su composición específica la avifauna estudiada se relacionó con las avifaunas de la cuenca del Balsas. La selva baja del cañón del Sabino es prioritaria para la conservación debido a su riqueza en especies endémicas y a la presencia una colonia reproductora de la guacamaya verde, Ara militaris, especie globalmente amenazada.
\end{abstract}

Palabras clave: avifauna, cañada del río Sabino, México, selva baja caducifolia, similitud específica, TehuacánCuicatlán.

\begin{abstract}
This study describes the birds of the tropical dry forest in Sabino Canyon, Oaxaca, in the southeastern part of the Tehuacán-Cuicatlán biosphere reserve. Field work was conducted between June 2005 and October 2006. A total of 113 species belonging to 13 orders and 34 families were recorded. Six species were classified as very abundant, 10 as abundant, 20 as common, 32 as uncommon, and 37 as rare; 79 species were permanent residents, 28 were winter visitors, 2 summer visitors, 3 transients, and 5 were local migrants; 15 species were classified as endemic to Mexico, and 23 are threatened. The bird composition of Sabino Canyon mainly resembles the avifauna the Balsas river basin. The avifauna of the tropical dry forest in Sabino Canyon is important for bird conservation due its richness in endemic birds and to the presence of an important reproductive colony of the Military Macaw, Ara militaris, a globally threatened bird species.
\end{abstract}

Key words: avifauna, Sabino Canyon, Mexico, tropical dry forest, specific similarity, Tehuacán-Cuicatlán.

\section{Introducción}

El bosque tropical caducifolio o selva baja caducifolia es un tipo de vegetación tropical caracterizado por un periodo corto de lluvias y un prolongado estío producto de su marcada estacionalidad climática, latitud y elevación. En este sistema la estacionalidad en la disponibilidad de agua es determinante para la estructura, fenología y

Recibido: 05 enero 2008; aceptado: 22 septiembre 2008 composición específica de la vegetación (Murphy y Lugo, 1986; Bullock y Solís-Magallanes, 1990), así como para la disponibilidad de recursos (Arizmendi y Ornelas, 1990; Poulin, 1993; Moya-Moreno, 2002). En México esta vegetación ocupa cerca del $12 \%$ de la extensión territorial (Trejo y Dirzo, 2000), aunque su cobertura disminuye rápidamente debido a las actividades humanas (Burgos y Mass, 2004).

Desde el punto de vista ornitológico, la selva baja ha sido considerada importante para la diversidad biológica 
mundial por su riqueza específica y abundancia, así como por su alta concentración de endemismos (Stotz et al., 1996). La avifauna de las selvas bajas representa cerca del 33\% de las especies terrestres de México y alrededor del 19\% de los endemismos para el territorio nacional (Escalante et al., 1998). De hecho es uno de los ambientes con mayor número de especies endémicas para México (GonzálezGarcía y Gómez de Silva, 2003), siendo particularmente la cuenca del Balsas considerada un área de endemismo de aves con relevancia mundial. (Endemic Bird Area: EBA 008, Stattersfield et al., 1998). Esta importancia biológica ha sido revelada principalmente a través de estudios ornitológicos descriptivos (p. e. Selander y Giller, 1959; Arizmendi et al., 1990; Ortiz-Pulido et al., 1995; FeriaArroyo, 2001; Ramírez-Albores y Ramírez-Cedillo, 2002; Almazán-Núñez y Navarro, 2006); sin embargo, mucho del conocimiento actual se basa en información limitada y general que deberá profundizarse (Vega et al., en prensa).

Estudios recientes -aunque no enfocados directamente a las selvas bajas- han permitido reconocer la influencia del aislamiento geográfico y la diversidad ambiental generada por la sierra Madre Occidental y el Eje Neovolcánico Transversal sobre la avifauna del oeste de México (Palomera-García et al., 1994; García-Trejo y Navarro, 2004). Una contribución notable es la de RíosMuñoz (2006) quien describe los patrones biogeográficos de riqueza específica y endemismo de las avifaunas en las selvas bajas de Mesoamérica, señalando la influencia de los eventos orográficos y climáticos en el establecimiento de 2 grupos de avifaunas: uno del sureste de México hasta el sur de Panamá y otro al oeste del istmo de Tehuantepec. De acuerdo con este trabajo, las zonas de mayor diversidad en las selvas secas se ubicaron en el istmo de Tehuantepec y el centro de Veracruz en México y en el Valle de Sula en Honduras; sin embargo, subrayó la necesidad de continuar con la descripción en zonas aún no estudiadas para mejorar el conocimiento al respecto que permita establecer estrategias adecuadas de conservación.

La avifauna del Valle de Tehuacán-Cuicatlán ha sido estudiada sólo parcialmente a pesar de la excepcional riqueza biológica de la región (Dávila et al., 2002) y los trabajos publicados referentes a su avifauna son escasos; Arizmendi y Espinosa de los Monteros (1996) estudiaron la avifauna de los ambientes áridos del Valle de Tehuacán, y Peterson et al. (2003) describieron la composición específica en los ambientes riparios de la Cañada de Cuicatlán, al sur de la región; sin embargo, para la avifauna de selva baja, que representa la tercera parte de la extensión total de la región, no existe ningún trabajo publicado. El presente estudio tiene como objetivo describir la comunidad de aves de la selva baja caducifolia en la cañada del río Sabino, Oaxaca, en la Reserva de la Biosfera Tehuacán-
Cuicatlán y comparar su similitud específica con avifaunas de otras regiones con àreas similares ocupadas por selva baja para generar una aproximación desde el punto de vista ecológico a la composición avifaunística de las selvas bajas de la reserva y su relación con otras selvas bajas de México.

\section{Materiales y métodos}

Área de estudio. La cañada del río Sabino se encuentra al noroeste del estado de Oaxaca, en el municipio de Santa María Tecomavaca, en la parte sureste de la Reserva de la Biosfera Tehuacán-Cuicatlán. La cañada del Sabino se formó por el paso del río del mismo nombre, alcanzando una profundidad de hasta $250 \mathrm{~m}$ desde su parte más alta (INEGI, 1981). Las coordenadas centrales del sitio son $17^{\circ} 51^{\prime} 57^{\prime}$ ' N, $97^{\circ} 01^{\prime} 50^{\prime}$ O y su altitud va de 580 a $850 \mathrm{~m} \mathrm{snm} \mathrm{(Fig.} \mathrm{1).} \mathrm{Su} \mathrm{vegetación} \mathrm{es} \mathrm{de} \mathrm{selva} \mathrm{baja}$ caducifolia asociada a bosques de cactáceas columnares (Valiente-Banuet et al., 2000). El clima es árido $\left(\mathrm{BS}_{0}\right)$, con temperatura media anual de $22^{\circ} \mathrm{C}$ (Salazar, 2001), máximo promedio de $37.4^{\circ} \mathrm{C}$ (mayo) y mínimo promedio de $13.2^{\circ} \mathrm{C}$ (enero); la precipitación promedio es de $450 \mathrm{~mm}$ y la temporada de lluvias abarca de fines de mayo a principios de octubre (datos originales de la estación meteorológica de Quiotepec 1961-1996). La aridez de la zona es producto de la sombra orográfica que proyectan las sierras de Juárez y de Zongolica sobre la región (Villaseñor et al., 1990; Dávila y Herrera-MacBride, 1997). La geología del lugar se caracteriza por la presencia de rocas sedimentarias calizas y lutitas del Cretácico inferior y conglomerados del Terciario inferior (INEGI, 1981).

Trabajo de campo y de gabinete. Entre junio de $2005 \mathrm{y}$ octubre de 2006 se realizaron 13 visitas (de 4 a 7 días) al sitio de estudio en las que se establecieron 11 parcelas circulares permanentes con radio fijo de $25 \mathrm{~m}$ distribuidas en un recorrido de aproximadamente $3.5 \mathrm{~km}$ a través del gradiente altitudinal del sitio de estudio, conservando una distancia mínima de $250 \mathrm{~m}$ entre parcelas. Entre noviembre de 2005 y octubre de 2006 se realizaron 2 conteos por visita en cada parcela registrando las especies y abundancia de las aves observadas en un lapso de 10 minutos, así como las especies observadas fuera y entre parcelas (Hutto et al., 1986). En cada salida se realizaron además observaciones focales desde promontorios elevados al borde del cañón (modificado de Ralph et al., 1994), registrando las especies observadas y su abundancia por un lapso de 3 horas después del amanecer y 3 antes del atardecer. Estos conteos permitieron registrar las aves de vuelo alto que no es posible observar en el interior de la selva dentro de los conteos de radio fijo. Además, en 12 salidas se utilizaron 


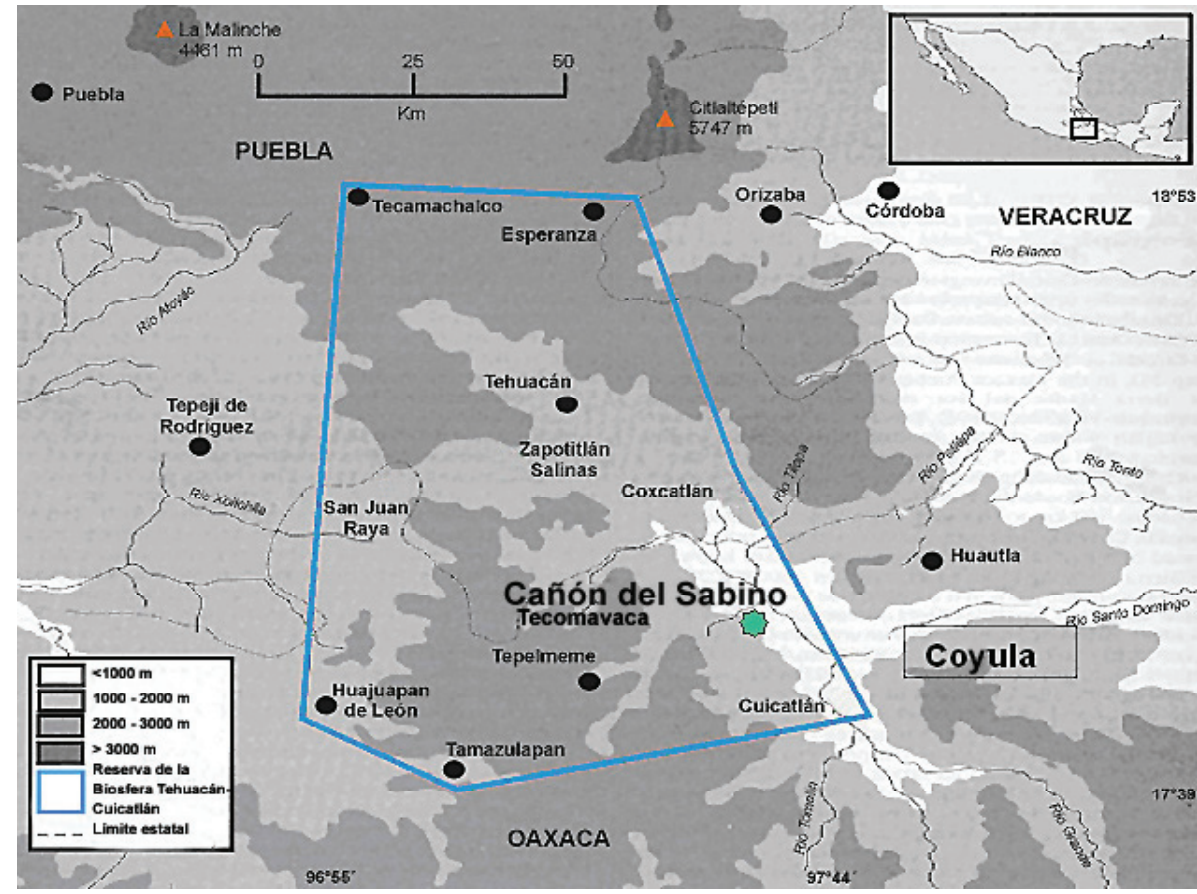

Figura 1. Ubicación del cañón del Sabino, Santa María Tecomavaca, Oaxaca. El sitio se encuentra en la parte sur de la Reserva de la Biosfera Tehuacán-Cuicatlán (modificado de Dávila et al., 2002).

89\%), común (31-64\%), poco común $(10-30 \%)$, rara $(1-9 \%)$. En los grupos donde la identificación específica es difícil, la estimación de abundancia se calculó por género (Empidonax, Myiarchus, Tyrannus (excepto Tyrannus crassirostris)). Para analizar la similitud y los patrones de composición avifaunística entre el Valle de Tehuacán-

redes de niebla (de 6 a 10 redes de 2.6 x 6, 9 y 12 m., con 24 mm de luz de malla), totalizando 3354 horas/red. Las aves capturadas en redes fueron identificadas, fotografiadas y posteriormente liberadas. Las observaciones y el trabajo de captura se suspendieron en condiciones de lluvia, dado que la actividad de las aves desciende sensiblemente en esas condiciones (Bibby et al., 2000). Las observaciones se realizaron con binoculares (10x35) y se usaron guías de campo para su identificación (Robbins et al., 1966; Pyle et al., 1987; Peterson y Chalif, 1989; Howell y Webb, 1995; National Geographic, 1999).

El listado sistemático se organizó de acuerdo con los nombres y los criterios de la AOU (2006) y se definieron las especies incluidas dentro de alguna categoría de amenaza de acuerdo a la NOM-059-SEMARNAT-2001 (DOF., 2002), IUCN (2006) y CITES (2005); el estatus de residencia se determinó considerando los criterios de Howell y Webb (1995) y el estatus de endemismo de acuerdo con GonzálezGarcía y Gómez de Silva (2003). Se aplicaron los modelos de acumulación de especies de Jacknife-1 y MichaelisMenten usando el programa EstimateS (Colwell, 2006) para tener una predicción de la riqueza específica total de la comunidad. Para obtener un índice de la importancia de las especies registradas para el ensamble de aves en la zona de estudio se calculó la abundancia relativa de las aves a través de la expresión porcentual de la frecuencia relativa (Pettingill, 1970), considerando las siguientes categorías: muy abundante (90-100\%), abundante (65-
Cuicatlán y otras regiones del país que representan extensiones importantes de selva baja se realizó un análisis de conglomerados (Trejo-Torres y Ackerman, 2002) utilizando los listados disponibles en la base de datos de las AICAS ( Áreas de importancia para Ia conservación de las aves; Arizmendi y Márquez-Valdelamar, 2000). Se seleccionaron los listados más completos y confiables (Gómez de Silva y Medellín, 2001) de las áreas que de acuerdo con la base de datos tienen una proporción de selva baja mayor o igual a la registrada en la Reserva de la Biosfera Tehuacán-Cuicatlán (29\%), el AICA donde se ubica la cañada del Sabino. Los registros de éste trabajo y los obtenidos por Peterson et al. (2003) se adicionaron al listado del AICA Tehuacán-Cuicatlán. Para evaluar la similitud específica entre las AICAS seleccionadas sólo se consideraron las especies terrestres residentes, excluyendo las introducidas (Columba livia, Passer domesticus, Sturnus vulgaris), debido a que su presencia depende de la actividad humana (McKinney, 2002), así como las migratorias, debido a que su presencia puede deberse más bien a factores históricos y geográficos que a factores ecológicos en el hábitat disponible en una región (Komar, 2003). Una vez depurada la lista de aves obtenida (424 sp.), se construyó una base de datos de presencia-ausencia para las 17 AICAS seleccionadas y se realizó un análisis de conglomerados (coeficiente de similitud de Jaccard, ligamiento completo) usando el programa Biodiversity Pro (McAlecee, 1997). 


\section{Resultados}

Descripción de la avifauna. Se registraron 113 especies de aves, pertenecientes a 13 órdenes y 34 familias (Apéndice 1). Las familias mejor representadas fueron Tyrannidae (21 especies), Parulidae (13 especies), Trochilidae ( 7 especies) y Cardinalidae (7 especies). Entre las aves registradas se encuentran especies afines a la vertiente del Pacífico (p. ej. Momotus mexicanus, Ortalis poliocephala, Pheucticus chrysopeplus y Amazilia violiceps); a la cuenca del río Balsas (p. ej. Cynanthus sordidus, Melanerpes hypopolius, Xenotriccus mexicanus y Campylorhynchus jocosus); a la vertiente del Atlántico (p. ej. Euphonia hirundinacea, Thraupis abbas y T. episcopus), así como de los ambientes montanos del interior del país (p. ej. Atthis heloisa y Ptilogonys cinereus) y de las regiones áridas del Altiplano mexicano (p. ej. Picoides scalaris, Mimus polyglottos, Lanius ludovicianus y Carpodacus mexicanus). De acuerdo con el modelo predictivo de Jacknife-1, se registró el 71\% de la avifauna, mientras que el modelo de MichaelisMenten indica que se registró el 82\% (Fig. 2).

Según la estimación de abundancia relativa, $6(5.3 \%)$ de las especies fueron muy abundantes, 7 (6.1\%) abundantes, $20(17.6 \%)$ comunes, $32(28.3 \%)$ poco comunes, 37 $(32.7 \%)$ raras y para $11(9.7 \%)$ de las especies el valor de abundancia no pudo ser determinado (géneros Empidonax, Myiarchus y Tyrannus). De las especies registradas, 79 (69.9\%) fueron catalogadas como residentes, 28 como visitantes invernales (24.7\%), 2 como visitantes de verano $(1.7 \%)$ y 3 como transitorias $(2.6 \%)$. No se consideraron los colibríes del género Calothorax porque en la región hay 2 especies del género ( $C$. lucifer y $C$. pulcher) con diferentes estatus de residencia (visitante invernal

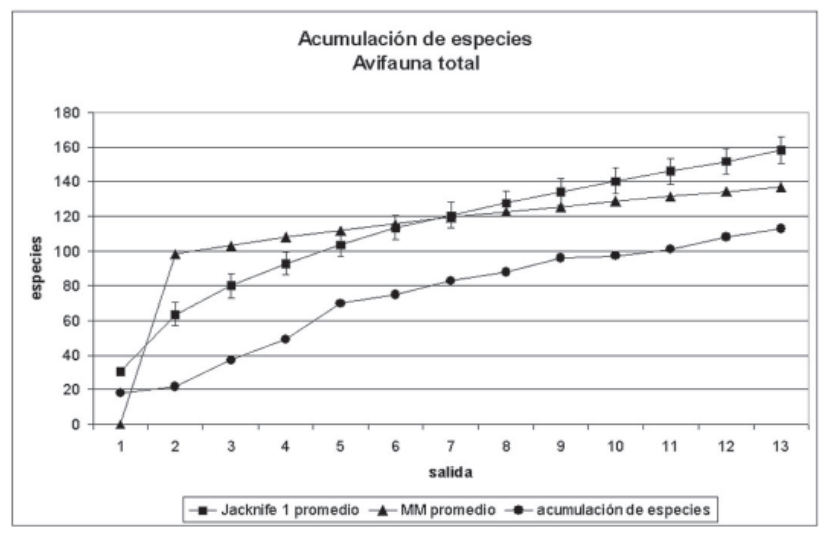

Figura 2. Curva de acumulación de especies y predicción del tamaño total de la avifauna de acuerdo con los modelos de Jacknife-1 $( \pm \mathrm{DE})$ y Michaelis-Menten. Se han registrado el 71y $82 \%$ de las especies, respectivamente. y residente reproductor, respectivamente). De las 33 especies migratorias (Howell y Webb 1995) 26 tuvieron una abundancia relativa de poco común a rara (Apéndice 1), mientras que 5 especies consideradas residentes permanentes en la literatura especializada (Leptotila verreauxi, Xenotriccus mexicanus, Vireo hypochryseus, Polioptila albiloris y Basileuterus rufifrons) se registraron sólo durante el periodo de primavera/verano (marzo a octubre), por lo que se les consideró como residentes con movimientos estacionales. Del total de especies registradas, $15(13.2 \%)$ son endémicas de México: 7 del oeste de México, 6 de la cuenca del río Balsas y 2 de los ambientes montanos del centro del país (Apéndice 1), mientras que 23 de las especies registradas $(20.3 \%)$ se ubicaron en alguna categoría de amenaza, según las disposiciones nacionales o internacionales consideradas (Apéndice 1); de acuerdo con estos criterios Ara militaris y Falco peregrinus son las especies que se encuentran en mayor riesgo de extinción. Relación con otras regiones de México con selvas bajas. El análisis de clasificación generó 2 agrupaciones avifaunísticas principales (Fig. 3). Los valores de similitud entre todas las AICAS se registran en el Apéndice 2. La primera asociación incluyó las avifaunas de AICAS relacionadas con el occidente en 3 subgrupos, además de las islas Marías. El primer subgrupo se formó por las avifaunas afines a la cuenca del río Balsas (Tehuacán-Cuicatlán, Sierra de Huautla y Cuenca Baja del Balsas) que incluyeron aves comunes en las selvas bajas de la vertiente Pacífica (como Ortalis poliocephala, Caprimulgus ridgwayi, Momotus mexicanus, Vireo hypochryseus y Turdus rufopalliatus entre otros); además, las AICAS de Tehuacán-Cuicatlán y Huautla incluyen especies endémicas, como Melanerpes hypopolius, Cynanthus sordidus, Xenotriccus mexicanus, Campylorhynchus jocosus, Pipilo albicollis y Aimophila mystacalis. El segundo subgrupo se formó por avifaunas con influencia de la sierra Madre Occidental (Tancítaro, Coalcomán-Pómaro y Álamos-Río Mayo), que incluyeron especies típicas de bosques templados, como Rhynchopsitta pachyrhyncha, Trogon mexicanus y Glaucidium gnoma. El tercer subgrupo incluye las avifaunas de la costa del Pacífico (Manialtepec, Marismas Nacionales, Chamela-Cuitzmala) con especies como Deltarhynchus flammulatus, Forpus cyanopygius y Chlorostilbon auriceps. El segundo grupo incluyó las avifaunas del sureste del país y de la península de Yucatán. Las AICAS del sureste del país (La Sepultura, Centro de Veracruz, Zapotal-Mactumatza y La Mancha) compartieron especies neotropicales, como Amazona autumnalis, Heliomaster longirostris, Momotus momota, Eumomota superciliosa, Thraupis abbas y T. episcopus; mientras que las AICAS de la península de Yucatán (Los Petenes, Dzilám, e Ichka' Ansijo) compartieron además especies endémicas, como Amazona xantholora, Meleagris 


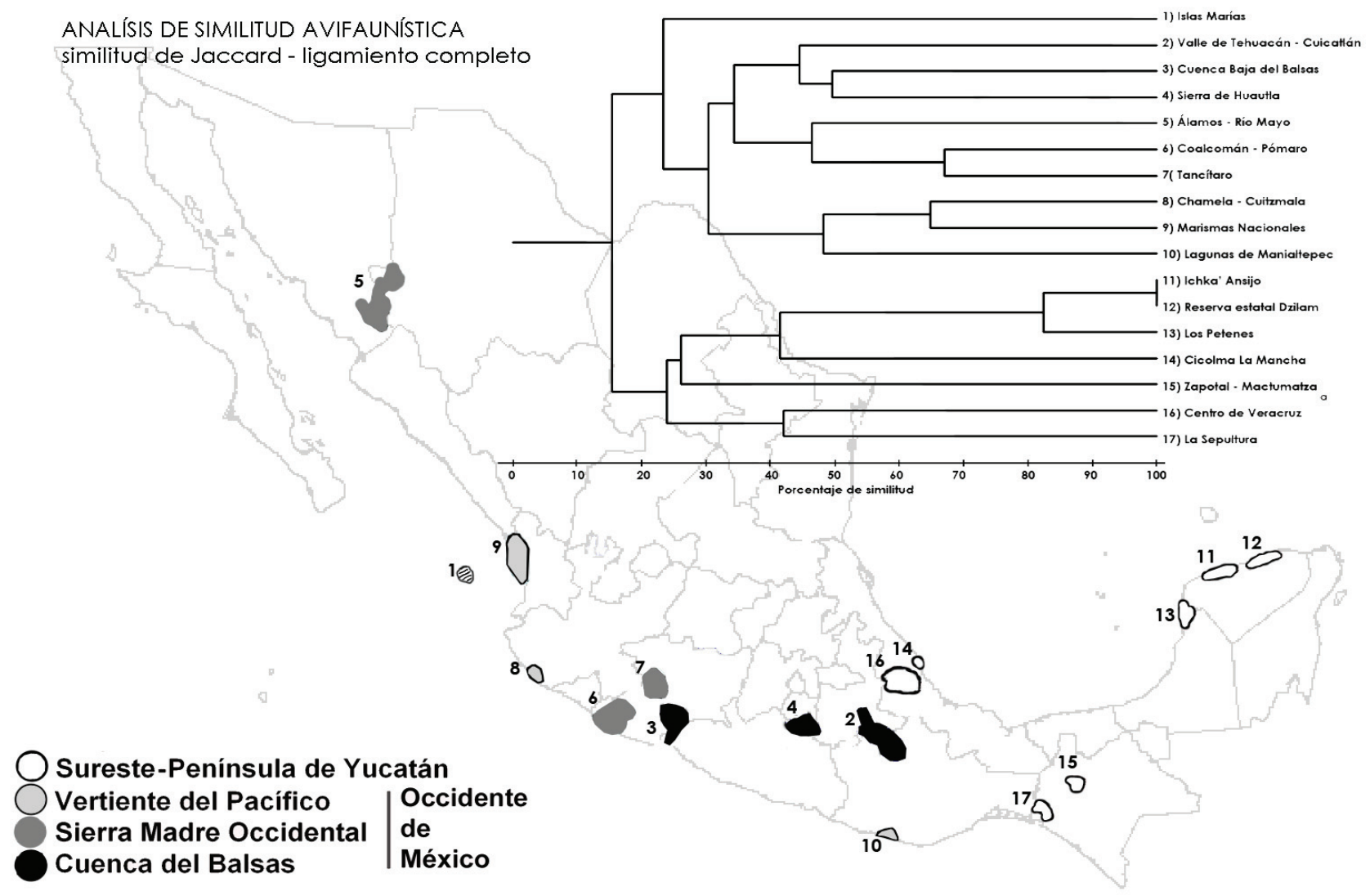

Modificado de CONABIO

http://conabioweb.conabio.gob.mx/aicas/doctos/aicasmapa.html

Figura 3. Análisis de similitud avifaunística de algunas regiones de México (AICAS) con selva baja caducifolia. Se utilizó el índice de Jaccard y ligamiento completo entre grupos. La avifauna del AICA Tehuacán-Cuicatlán se relaciona con las AICAS de la cuenca del río Balsas (mapa modificado de CONABIO, 2007).

ocellata, Campylorhynchus yucatanicus y Cyanocorax yucatanicus.

\section{Discusión}

Descripción de la avifauna. De acuerdo con los modelos predictivos de la riqueza total de la avifauna, aún queda por registrar entre el 29\% (Jacknife) y el 18\% (MichaelisMenten) de las aves que se distribuyen en la zona de estudio. Tomando en cuenta que no se incluyeron muestreos dirigidos a especies nocturnas ni acuáticas, consideramos que el listado obtenido es útil para la descripción de la avifauna en el sitio, pues incluye la mayoría de las especies presentes, así como las más representativas de la comunidad (Gómez de Silva y Medellín, 2001).

El hecho de que sólo el $15.2 \%$ de los taxa registrados fueron abundantes o muy abundantes, mientras que los taxa poco comunes y raros representaron el $65.7 \%$ de la comunidad, coincide con los patrones generales de las comunidades (Begon et al., 1999), y es notable la cantidad de especies migratorias con valores de abundancia de rara a poco común ( 26 de 33 , es decir el $78.7 \%$ ), lo que podría indicar cierta relación entre los patrones de abundancia de las aves y sus movimientos estacionales debidos a las fluctuaciones en la disponibilidad de recursos (Arizmendi y Ornelas, 1990; Poulin, 1993; Moya-Moreno, 2002). En ese sentido, el patrón de presencia estacional registrado para Leptotila verreauxi, Xenotriccus mexicanus, Vireo hypochryseus, Polioptila albiloris y Basileuterus rufifrons (residentes permanentes según Howell y Webb 1995) podría explicarse con probables movimientos estacionales de estas aves en respuesta a las variaciones de la selva seca (Poulin et al., 1993), ya sea que se trate de movimientos 
locales (desplazamientos de corta distancia entre ambientes contiguos) o altitudinales (desplazamientos a ambientes diferentes a través del rango altitudinal en el área de distribución) (Ornelas y Arizmendi, 1995). Este tipo de movimientos locales de aves residentes permanentes se ha registrado en las selvas bajas del istmo de Tehuantepec para Campylopterus hemileucurus, Phaethornis longirostris, Xiphorynchus flavigaster y Chlorostibon canivetti (Rodríguez-Contreras, 2004); en la selva baja de la región de Chamela, para Amazilia rutila, Phaethornis superciliosus (Arizmendi y Ornelas, 1990) y Amazona finschi (Renton, 2001) e inclusive para Ara militaris en el cañón del río Sabino (Rivera-Ortiz et al., 2008).

La avifauna estudiada está influida por componentes afines a las selvas bajas de la vertiente del Pacífico y a la cuenca del Balsas, debido a que dicha cuenca funciona como un corredor continuo de hábitat que conecta la costa occidental con el interior del país, incluyendo el sur de Puebla y el noroeste de Oaxaca, provocando que las comunidades avifaunísticas tengan composiciones similares (Vega et al., en prensa). La presencia de especies afines a la vertiente del Atlántico se explica por la cercanía geográfica de la zona de estudio con las sierras Madre, la Oriental y la del Sur (Morrone, 2005), fenómeno registrado previamente para los ambientes riparios de Tomellín y Quiotepec, cercanos a la zona de estudio (Peterson et al., 2003); mientras que la presencia de aves relacionadas con las zonas áridas del Altiplano en la selva baja de Tecomavaca (como Colaptes auratus, Lanius ludovicianus, Mimus polyglottos y Carpodacus mexicanus) se explica por el intercambio de especies entre las selvas bajas de la cañada de Cuicatlán y los ambientes más secos del Valle de Tehuacán, una zona árida que recibe influencia biológica de los desiertos de Norteamérica y el Altiplano mexicano (Dávila et al., 2002); del mismo modo, el intercambio de especies provoca que el Valle de Tehuacán incorpore aves características de selvas bajas, como Ortalis poliocephala y Momotus mexicanus (Arizmendi y Espinosa de los Monteros, 1996). La presencia de especies características de ambientes más elevados puede explicarse por el intercambio de organismos entre los ambientes contiguos (Navarro, 1992), como los bosques de encino y pino-encino de la sierra Norte de Oaxaca, movimientos que pueden ser provocados por fluctuaciones en la disponibilidad y abundancia de recursos alimenticios (Ornelas y Arizmendi, 1995; Vega et al., en prensa).

Endemismo y conservación. La elevada riqueza de especies endémicas en Tecomavaca se debe a que la biota de la región diversificó bajo la influencia del aislamiento geográfico y la diversidad ambiental provocados por el levantamiento de los sistemas montañosos de las sierras Madre Occidental, el Eje Neovolcánico Transversal y la sierra Madre del Sur (García-Trejo y Navarro, 2004; Ríos-Muñoz, 2006, Morrone, 2005). La sierra Madre del Sur desempeña un papel importante en la composición avifaunística de las selvas bajas del Valle de TehuacánCuicatlán, ya que las montañas del norte de Oaxaca representan el límite sur del Valle así como un corredor para las aves de las vertientes atlántica y pacífica, como Thraupis abbas, T. episcopus y Euphonia hirundinacea, registradas en Tecomavaca (García-Trejo y Navarro, 2004). Desde la óptica de la conservación, la riqueza de endemismos coloca la cañada del río Sabino en un sitio prioritario para la conservación de las aves (Peterson et al., 1993; Balmford, 2002; Mace y Collar, 2002; Arizmendi, 2003) y cabe mencionar que la zona de estudio se ubica en el área de influencia de la cuenca del Balsas, reconocida como una de las principales áreas de endemismo de aves en el mundo (Stattersfield et al., 1998). La presencia de una de las colonias de anidación de guacamaya verde más importantes en el país en el cañón del río Sabino (RiveraOrtiz et al., 2008) realza la necesidad de conservar los ambientes naturales en la región para proteger esta especie que está considerada por la IUCN como vulnerable en todo el mundo (Sánchez-Azofeifa et al., 1999; Arizmendi y Márquez-Valdelamar, 2000).

Relación con otras regiones de México con selvas bajas. Respecto al análisis de similitud avifaunística con otras regiones de selva baja en México, debe considerarse que los resultados se basan en listas que incluyeron tanto las especies registradas en selvas bajas como las registradas en los ambientes adyacentes y que sólo se consideraron las regiones donde la avifauna ha sido estudiada obteniendo listados completos. Además, los listados no necesariamente son uniformes en cuanto a su calidad por lo que sus similitudes pueden estar sesgadas por las diferencias en el tamaño de las avifaunas. Lo anterior puede incidir sobre los valores de similitud calculados, pero fue posible hacer inferencias con sentido biológico sobre el patrón detectado. La asociación entre las avifaunas afines a la vertiente del Atlántico en un grupo separado de las del occidente del país se explica porque las condiciones ecológicas actuales de las selvas bajas de ambas regiones tienen historias diferentes. En primer lugar, las selvas bajas del Atlántico se originaron en ambientes con baja elevación sobre el nivel del mar y condiciones orográficas que favorecieron que las avifaunas del sureste de México y de la península de Yucatán se relacionaran biogeográficamente con los bosques tropicales de Centroamérica (Ríos-Muñoz, 2006); este hecho queda en evidencia por la presencia de especies eminentemente neotropicales como Amazona autumnalis, Heliomaster longirostris, Momotus momota, Eumomota superciliosa, Thraupis abbas y T. episcopus. De manera notable, las avifaunas de la península de 
Yucatán quedaron bien separadas del resto de avifaunas del sureste debido a que la baja altitud sobre el nivel del mar y la falta de relieves considerables en la península provocan que los vientos y ciclones del Caribe mantengan aislada la biota peninsular de la influencia climática y ecológica del continente, (Morrone, 2005) por lo que en la región se encuentran aves endémicas de distribución restringida como Amazona xantholora, Meleagris ocellata, Campylorhynchus yucatanicus y Cyanocorax yucatanicus (Ríos-Muñoz, 2006). Por otro lado, la distribución de las selvas bajas del occidente de México tiene su origen en el aislamiento producido por el surgimiento de la sierra Madre Occidental y el Eje Neovolcánico Transversal (Becerra, 2005) y la sierra Madre del Sur (Morrone, 2005). Dicho aislamiento originó los endemismos característicos de las selvas bajas del Pacífico y del Balsas, tanto en aves (RíosMuñoz, 2006) y reptiles (García, 2006) como en plantas (género Bursera; Rzedowski, 1978; Becerra, 2005).

Particularizando en las asociaciones entre las avifaunas del occidente, encontramos en primer lugar las selvas bajas de la vertiente del Pacífico. Estas avifaunas estuvieron asociadas debido a que la selva baja representa un ambiente prácticamente ininterrumpido a través de la costa occidental, lo que favorece que avifaunas geográficamente distantes se asemejen a lo largo de la distribución de la selva baja en el oeste de México y compartan especies de aves endémicas como Deltarhynchus flammulatus, Forpus cyanopygius y Chlorostilbon auriceps (Vega et al., en prensa). Por otro lado, la asociación entre las áreas de la sierra Madre Occidental se debe a que sus avifaunas reciben influencia de los ambientes elevados de este sistema montañoso. Las AICAS de éste subgrupo tienen del 23 al $58 \%$ de su extensión cubierta por bosques de coníferas y encinos (Arizmendi y MárquezValdelamar, 2000) y la especie que compartieron las 3 AICAS (Glaucidium gnoma) es un ave típica de bosques de coníferas y encinos (García-Trejo y Navarro, 2004). Finalmente, la asociación entre las avifaunas de la cuenca del Balsas se debe a que ésta representa un continuo de selvas bajas aisladas por la influencia de la sierra Madre Occidental, la sierra Madre del Sur y el Eje Neovolcánico, favoreciendo que las avifaunas de la región compartieran especies propias de las selvas bajas de la vertiente Pacífica, así como la evolución de numerosas especies endémicas. Esta influencia se manifiesta desde la desembocadura del Balsas en el Pacífico entre Michoacán y Guerrero, hasta el interior del territorio en los estados de Morelos, el sur de Puebla y el norte de Oaxaca (Becerra, 2005; Ríos-Muñoz, 2006) y debido a ella, las comunidades incluyen especies comunes de las selvas del Pacífico, mientras el Valle de Tehuacán-Cuicatlán y la sierra de Huautla incluyen aves endémicas de la cuenca alta del Balsas, como Melanerpes hypopolius, Xenotriccus mexicanus, Campylorhynchus jocosus y Aimophila mystacalis (Stattersfield et al., 1998; Ríos-Muñoz, 2006).

De acuerdo con los resultados obtenidos, el cañón del Sabino representa una área de importancia primaria para la conservación de las aves en México debido a su notable riqueza en especies endémicas y la colonia reproductora de Ara militaris que alberga. Sin embargo debe subrayarse que el conocimiento acerca de las aves de la región no estará completo hasta que se realicen estudios avifaunísticos que cubran los ambientes de la Reserva que aún no han sido descritos desde el punto de vista ornitológico.

\section{Agradecimientos}

Agradecemos a los señores Efraín Hernández, Gregorio Cruz y demás autoridades comunales de Santa María Tecomavaca, por las facilidades brindadas para la realización del estudio. A dos revisores anónimos que mejoraron sustancialmente la presentación del manuscrito con sus comentarios. A los M. en C. Patricia Ramírez y César Ríos por las sugerencias útiles para la realización del trabajo. A todas las personas que nos apoyaron en el trabajo de campo: Ricardo Álvarez, Eric Balderas, Ana María Contreras, Pedro Estrella, Arnoldo Flores, Ivonne Flores, Verónica García, Osvaldo Hernández, Araceli Jaimes, Víctor Jiménez, Jorge Mendoza, Miguel Morales, Enrique Ortega, Jesús Ortega, Elisa Parra, Francisco Alberto Rivera, Claudia Rodríguez, Carlos Soberanes, Manuel Soberanes, Alejandro Vázquez y Adolfo Vital. Este trabajo se realizó en el Laboratorio de Ecología de la UBIPRO, FES Iztacala, UNAM, con el apoyo de los proyectos CONABIO-DS003, DT006 y PAPIIT-DGAPAUNAM IN-207305 a cargo de la Dra. Arizmendi. Los estudios profesionales de Leopoldo Vázquez fueron apoyados por una beca del programa PAEA-UNAM, en la FES Iztacala.

\section{Literatura citada}

Almazán-Núñez, R. C. y A. G. Navarro. 2006. Avifauna de la subcuenca del río San Juan, Guerrero, México. Revista Mexicana de Biodiversidad 77:103-114.

AOU(American Ornithologists' Union). 2006. List of the 2041 bird species (with scientific and English names) known from the A.O.U. checklist area. www.aou.org/aou/birdlist.html; consulta: 21 de septiembre de 2006.

Arizmendi, M. C. 2003. Estableciendo prioridades para la conservación de las aves. In Conservación de aves: experiencias en México, H. Gómez de Silva y A. Oliveras de Ita (eds.). CIPAMEX/CONABIO/NFWF, México, D. F. 
p. 133-149.

Arizmendi, M. C. y A. Espinosa de los Monteros. 1996. Avifauna de los bosques de cactáceas columnares del Valle de Tehuacán, Puebla. Acta Zoológica Mexicana (ns) 67:25-46.

Arizmendi, M. C. y J. F. Ornelas. 1990. Hummingbirds and their floral resources in a tropical dry forest in México. Biotropica 22:172-180.

Arizmendi, M. C. y L. Márquez-Valdelamar. 2000. Áreas de importancia para la conservación de las aves en México. CIPAMEX, México, D. F. 440 p.

Arizmendi, M. C., H. Berlanga, L. M. Márquez-Valdelamar, L. Navarijo y J. F. Ornelas. 1990. Avifauna de la región de Chamela, Jalisco, Cuadernos 4. Instituto de Biología, UNAM, México, D. F. 62 p.

Balmford, A. 2002. Selecting sites for conservation. In Conserving bird biodiversity. Principles and their applications, K. Norris y D. J. Pain (eds.). Cambridge University Press, Cambridge. p. 74-104.

Becerra, J. X. 2005. Timing the origin and expansion of the dry Mexican forest. Proceedings of the National Academy of Science 102:10919-10923.

Bibby, C. J., N. D. Burgess, D. A. Hill y S. Mustoe. 2000. Bird census techniques, segunda edición. Academic, London. $302 \mathrm{p}$.

Bullock, S. H., J. A. Solís-Magallanes. 1990. Phenology of canopy trees of a tropical deciduous forest in Mexico. Biotropica 22:22-35.

CITES (Convención sobre el Comercio Internacional de Especies Amenazadas de Flora y Fauna Silvestres). 2005. Apéndices I, II y III. www.cites.org/; consulta: 18 de mayo de 2005.

Colwell, R. K. 2006. Estimate S: Statistical estimation of species richness and shared species from samples. Version 8. http:// viceroy.eeb.uconn.edu/EstimateS; consulta: 29 de enero de 2007.

Comisión para el Conocimiento y Uso de la Biodiversidad. 2007. Mapa de Áreas de Importancia para la Conservación de las Aves http://conabioweb.conabio.gob.mx/aicas/doctos/ aicasmapa.html; consulta:11 de febrero de 2007.

Dávila, P. y O. Herrera-MacBride. 1997. México: CPD site MA4. Tehuacán-Cuicatlán region, México. In Centers of plant diversity: a guide and strategy for their conservation, S. D. Davis,, V. H. Heywood, O. Herrera-MacBride, J. Villalobos y A. C. Hamilton (eds.), WWF/ IUCN, Gland. p. 139-143.

Dávila, P., M. C. Arizmendi, A. Valiente-Banuet, J. L. Villaseñor, A. Casas y R. Lira. 2002. Biological diversity in the Tehuacán-Cuicatlán Valley, México. Biodiversity and Conservation 11:421-442.

DOF. 2002. Norma Oficial Mexicana NOM-059-SEMARNAT2001. Protección ambiental-Especies nativas de México de flora y fauna silvestres-Categorías de riesgo y especificaciones para su inclusión, exclusión o cambio-Lista de especies en riesgo. Diario Oficial de la Federación, 6 de marzo 2002, p. 1-153. México.

Escalante, P., A. G. Navarro-Sigüenza y A. T. Peterson. 1998. Un análisis geográfico, ecológico e histórico de la diversidad de aves terrestres de México. In Diversidad biológica de México. Orígenes y distribución, T. P. Ramamoorthy, R.
Bye, A. Lot, J. Fa. (eds.). Instituto de Biología. UNAM, México, D. F. p. 279-304.

Feria Arroyo, T. P. 2001. Patrones de distribución de las aves residentes de la cuenca del Balsas. Tesis maestría, Posgrado en Ciencias Biológicas, Facultad Ciencias, Universidad Nacional Autónoma de México, México, D. F. 83 p.

García, A. 2006. Using ecological niche modelling to identify diversity hotspots for the herpetofauna of Pacific lowlands and adjacent interior valleys of México. Biological Conservation 130:25-46.

García-Trejo, E. A. y A. G. Navarro. 2004. Patrones biogeográficos de la riqueza de especies y el endemismo de la avifauna en el oeste de México. Acta Zoológica Mexicana 20:167-185.

Gómez de Silva, H. y R. A. Medellín. 2001. Evaluating completeness of species lists for conservation and macroecology: a case study of Mexican land birds. Conservation Biology 15:1384-1395.

González-García, F. y H. Gómez de Silva. 2003. Especies endémicas: riqueza, patrones de distribución y retos para su conservación. In Conservación de aves: experiencias en México, H. Gómez de Silva y A. Oliveras de Ita. (eds.). CIPAMEX/CONABIO/NFWF, México, D. F. p. 150-194.

Howell, S. N. G. y S. Webb. 1995. A guide to the birds of Mexico and northern Central America. Oxford University Press, New York. 823 p.

Hutto, R. L., S. M. Pletschet y P. Hendricks. 1986. A fixedradius point count method for nonbreeding and breeding season use. The Auk 103:593-602.

INEGI (Instituto Nacional de Estadística, Geografía e Informática). 1981. Carta Geológica E14-9 Oaxaca. Escala 1:250 000. México, D. F.

IUCN (Unión Internacional para la Conservación de la Naturaleza y de los Recursos Naturales). 2006. The World Conservation Union Red List of Threatened Species. www.iucnredlist.org; consulta: 20 de diciembre de 2006.

Begon, M., J. L. Harper, C. R. Townsend. 1999. Ecología: individuos, poblaciones y comunidades. Omega, Barcelona. $1148 \mathrm{p}$.

Komar, O. 2003. En las listas faunísticas, no todas las especies son iguales: las aves de El Salvador como un ejemplo. In Conservación de aves: experiencias en México. H. Gómez de Silva y A. Oliveras de Ita (eds.). CIPAMEX/CONABIO/ NFWF, México, D. F. p. 57-61.

Mace, G. M. y N. J. Collar. 2002. Priority-setting in species conservation. In Conserving Bird Biodiversity. General principles and their application, K. Norris, D. J. Pain (eds.). Cambridge University Press, Cambridge. p. 61-73.

McAleece, N. 1997. Biodiversity. Profesional. Ver. 2. The Natural Hystory Museum and The Scottish Association for Marine Science. http://www.sams.ac.uk/

Mckinney, M. L. 2002. Urbanization, biodiversity and conservation. BioScience 52:883-890.

Morrone, J. J., D. Espinosa J. Llorente-Bousquets. 2002. Mexican biogeographic provinces: preliminary scheme, general characterizations, and synonymies. Acta Zoológica Mexicana 85:83-108.

Morrone, J. J. 2005. Hacia una síntesis biogeográfica de México. Revista Mexicana de Biodiversidad 76:207-252. 
Moya Moreno, H. 2002. Disponibilidad de alimento y estructura del hábitat en la distribución y abundancia de aves insectívoras en una selva baja en Estipac, Jalisco. Tesis maestría, Posgrado en Ciencias Biológicas, Facultad de Ciencias, UNAM, México, D. F. 95 p.

Murphy, P. G., A. E. Lugo. 1986. Ecology of the tropical dry forest. Annual Review of Ecology and Systematics 17:6788.

National Geographic Society. 1999. Field guide to the birds of North America, tercera edición. National Geographic Society, Washington, D.C. 464 p.

Navarro Sigüenza, A. G. 1992. Altitudinal distribution of birds in the Sierra Madre del Sur, Guerrero, México. The Condor 94:29-39.

Ornelas, J. F. y M. C. Arizmendi. 1995. Altitudinal migration: implications for the conservation of the Neotropical migrant avifauna of western México. In Conservation of Neotropical migratory birds in México, M. H. Wilson y S. A. Sader. (eds.). Symposium-Workshop. Los Tuxtlas, Veracruz, México. UNAM, U. Maine, USFWS/NBS. Maine Agricultural and Forest Experiment Station. Miscellaneous Publication.. p. 98-112.

Ortiz-Pulido, R., H. Gómez de Silva, F. González-García y A. Álvarez. 1995. Avifauna del centro de investigaciones costeras La Mancha, Veracruz, México. Acta Zoológica Mexicana (ns) 66:87-118.

Palomera-García, C., E. Santana, R. Amparán-Salido. 1994. Patrones de distribución de la avifauna en tres estados del occidente de México. Anales de Instituto de Biología, Universidad Nacional Autónoma de México, Serie Zoológica 65:137-175.

Peterson, A. T., G. Escalona-Segura, K. Zyskowski, D. A. Kluza y B. E. Hernández-Baños. 2003. Avifaunas of two dry forest sites in northern Oaxaca, México. Huitzil 4:3-9. www.huitzil.net.

Peterson, A. T., O. A. Flores-Villela, L. S. León-Paniagua, J. E. Llorente-Bousquets, M. A. Luis-Martínez, A. G. NavarroSigüenza, M. G. Torres-Chávez y I. Vargas-Fernández. 1993. Conservation priorities in México: moving up in the World. Biodiversity Letters 1:33-38.

Peterson, T. y R. Chalif. 1989. Guía de aves de México. Diana, México, D. F. 473 p.

Pettingill, O. S. 1970. Ornithology in laboratory and field, cuarta edición. Burgess, Minneapolis, Minnesota. 470 p.

Poulin, B. y G. Lefebvre, R. McNeil. 1993. Variations in bird abundance in tropical arid and semi-arid habitats. Ibis 135:432-441.

Pyle, P., S. N. G. Howell, R. P. Yukick y D. F. De Sante. 1987. Identification guide to North American passerines. Slate Creek, Bolinas, Califoria. 278 p.

Ralph, C. J., G. R. Geupel, P. Pyle, T. E. Martin, D. F. De Sante y B. Milá. 1994. Manual de métodos de campo para el monitoreo de aves terrestres. United States Department of Agriculture. Forest Service. Pacific Southwest Research Station. General technical report PSW-GRT-159-Web. 46 p.

Ramírez-Albores, J. E. y M. G. Ramírez-Cedillo. 2002. Avifauna de la región oriente de Huautla, Morelos, México. Anales del Instituto de Biología, Universidad Nacional Autónoma de México, serie zoología 73:91-111.

Renton, K. 2001. Lilac-crowned parrot diet and food resource availability: resource tracking by a parrot seed predator. The Condor 103: 62-69.

Ríos Muñoz, C. A. 2006. Patrones biogeográficos de la avifauna de las selvas secas de Mesoamérica. Tesis maestría, Posgrado en Ciencias Biológicas, Facultad de Ciencias, UNAM, México, D.F. 73 p.

Rivera-Ortiz, F. A., Contreras-González A. M., SoberanesGonzález C., Valiente-Banuet A. y M. C. Arizmendi. 2008. Seasonal abundance and breeding chronology of the Military Macaw (Ara militaris) in a semi-arid region in Central Mexico. Ornitología Neotropical 19:255-263.

Robbins., S. C. B. Bruun y H. S. Zim. 1966. A guide to field identification birds of North America. Golden, Houston, Texas. $340 \mathrm{p}$.

Rodríguez Contreras, V. 2004. Distribución de las aves en Nizanda, Istmo de Tehuantepec, Oaxaca. Tesis, Facultad de Ciencias, UNAM, México, D. F. 56 p.

Rzedowski, J. 1978. Vegetación de México. Limusa, México, D. F. 423 p.

Salazar, J. M. 2001. Registro de guacamaya verde (Ara militaris) en los cañones del río Sabino y río Seco, Santa María Tecomavaca, Oaxaca, México. Huitzil 2:18-20.

Sánchez-Azofeifa, G. A., C. Quesada-Mateo, P. GonzálezQuesada, S. Dayanandan y K. S. Bawa. 1999. Protected areas and conservation of biodiversity in the tropics. Conservation Biology 13:407-411.

Selander, R. y D. R. Giller. 1959. The avifauna of the Barranca de Oblatos, Jalisco, México. The Condor 61:210-222.

Stattersfield, A. J., M. J. Crosby, A. J. Long y D. C. Wege. 1998. Endemic bird areas of the world. Priorities for biodiversity conservation. Birdlife International, Cambridge. p. 112113.

Stotz, D. F., J. W. Fitzpatrick, T. A. Parker III y D. K. Moskovits. 1996. Neotropical birds. Ecology and conservation. The University of Chicago Press, Illinois. 478 p.

Trejo, I., R. Dirzo. 2000. Deforestation of seasonally dry tropical forest: a nacional and local analysis in México. Biological Conservation 94:133-142.

Trejo-Torres, J. C., J. D. Ackerman. 2002. Composition patterns of Caribbean limestone forests: Are parsimony, classification, and ordination analyses congruent? Biotropica 34:502-515.

Valiente-Banuet, A., A.Casas, A. Alcántara, P. Dávila, N. FloresHernández, M. C. Arizmendi, J. L. Villaseñor y J. Ortega. 2000. La vegetación del Valle de Tehuacán-Cuicatlán. Boletín de la Sociedad Botánica de México 67:24-74.

Vega, J.H., M.C. Arizmendi y L. Morales. En prensa. La avifauna de las selvas bajas del occidente de México. In Diversidad, amenazas y áreas prioritarias para la conservación de las selvas secas del Pacífico de México, G. Ceballos, E. Espinosa y M. Maass (eds.), en prensa. CONABIO/WWF, México, D.F. p. 77-98.

Villaseñor, J. L., P. Dávila y F. Chiang. 1990. Fitogeografía del Valle de Tehuacán-Cuicatlán. Boletín de la Sociedad Botánica de México 50:135-149. 
Apéndice 1. Listado sistemático de la avifauna registrada en la selva baja caducifolia del cañón del Sabino, Santa María Tecomavaca, Oaxaca (AOU 2006).

Abundancia: $\mathrm{MA}=$ muy abundante; $\mathrm{A}=$ abundante, $\mathrm{C}=$ común $\mathrm{PC}=$ poco común $\mathrm{R}=$ rara. Estacionalidad: $\mathrm{RR}=$ residente reproductor; $\mathrm{VI}=$ visitante invernal; $\mathrm{VV}=$ visitante de verano; $* \mathrm{RR}=$ Especies con estatus de residentes permanentes que presentan un patrón de presencia estacional de acuerdo a los registros; TR = transitorio. Categoría de riesgo: $\mathrm{PR}=$ sujeta a protección especial; $\mathrm{P}$ = en peligro de extinción; $\mathrm{A}$ = amenazada; $\mathrm{II}$ = tráfico regulado; $\mathrm{I}$ = tráfico prohibido; $\mathrm{VU}$ = vulnerable; $\mathrm{NT}=$ casi amenazada.

\begin{tabular}{ccccc}
\hline Taxón & & \multicolumn{2}{c}{ Categoría de riesgo } \\
Abundancia & Estacionalidad & Endemismo & NOM-059 & IUCN
\end{tabular}

GALLIFORMES

\section{CRACIDAE}

Ortalis poliocephala

PC

RR

Oeste de México

LC

CICONIFORMES

\section{CATHARTIDAE}

Coragyps atratus

C

RR

Cathartes aura

A

RR

FALCONIFORMES

ACCIPITRIDAE

Elanus leucurus

Accipiter striatus

Accipiter cooperii

Buteogallus anthracinus

Buteogallus urubitinga

Buteo jamaicensis

FALCONIDAE

Herpetotheres cachinnans

Falco sparverius

Falco peregrinus

COLUMBIFORMES

COLUMBIDAE

Zenaida asiatica

Zenaida macroura

Columbina inca

Columbina passerina

Columbina talpacoti

Leptotila verreauxi

PSITTACIFORMES

\section{PSITTACIDAE}

Ara militaris

*RR

CUCULIFORMES

\section{CUCULIDAE}

Piaya cayana
MA

$\mathrm{R}$

PC

$\mathrm{R}$

PC

PC

C

C

RR

PC

PC

A

MA

C

MA

$\mathrm{R}$

PC

C

RR

RR

RR

RR

RR

RR
* RR

$\begin{array}{ccc}- & \text { LC } & \text { II } \\ \text { PR } & \text { LC } & \text { II } \\ \text { PR } & \text { LC } & \text { II } \\ \text { PR } & \text { LC } & \text { II } \\ \text { PR } & \text { LC } & - \\ - & \text { LC } & \text { II }\end{array}$

$\begin{array}{lll}- & \text { LC } & \text { II } \\ - & \text { LC } & \text { II } \\ \text { PR } & \text { LC } & \text { I }\end{array}$


Apéndice 1. Continúa

$$
\text { Taxón }
$$

Geococcyx velox

\section{STRIGIFORMES}

\section{TYTONIDAE}

Tyto alba

STRIGIDAE

Bubo virginianus

Glaucidium brasilianum

CAPRIMULGIFORMES

\section{CAPRIMULGIDAE}

Chordeiles minor

Caprimulgus ridgwayi

APODIFORMES

APODIDAE

Aeronautes saxatalis

TROCHILIDAE

Cynanthus sordidus

Cynanthus latirostris

Amazilia violiceps

Lampornis clemenciae

Calothorax sp.

Archilochus colubris

Atthis heloisa

TROGONIFORMES

TROGONIDAE

Trogon elegans

\section{CORACIIFORMES}

\section{MOMOTIDAE}

Momotus mexicanus

ALCEDINIDAE

Chloroceryle americana

\section{PICIFORMES}

\section{PICIDAE}

Melanerpes hypopolius

Picoides scalaris

Colaptes auratus

PASSERIFORMES

TYRANNIDAE
Abundancia

PC

Estacionalidad

RR

RR

PC

RR

MA

RR

R

TR

$\mathrm{R}$

RR

MA

RR

A

RR

$\mathrm{R}$

C

$\mathrm{R}$

PC

PC

PC

C

RR

C

$\mathrm{R}$

$\mathrm{RR}$

RR

Cuenca del Balsas

Oeste de México

$\mathrm{RR}$

RR

TR

RR

Ambientes montanos

Endemismo

Categoría de riesgo

NOM-059 IUCN CITES

LC

LC II

A $\quad$ LC $\quad$ II

LC II

LC

LC

LC

LC II

LC II

LC II

LC II

LC II

LC II

LC II

LC

Oeste de México

LC

LC

C RR

Cuenca del Balsas

LC

A $\quad$ RR

PC

RR

LC

LC 
Apéndice 1. Continúa

\begin{tabular}{|c|c|c|c|c|c|c|}
\hline Taxón & Abundancia & Estacionalidad & Endemismo & NOM-059 & $I U C N$ & CITES \\
\hline Camptostoma imberbe & $\mathrm{PC}$ & $\mathrm{RR}$ & - & - & $\mathrm{LC}$ & - \\
\hline Xenotriccus mexicanus & $\mathrm{C}$ & $* \mathrm{RR}$ & Cuenca del Balsas & PR & NT & - \\
\hline Contopus sordidulus & $\mathrm{R}$ & VV & - & - & $\mathrm{LC}$ & - \\
\hline Empidonax albigularis & ND & VI & - & - & $\mathrm{LC}$ & - \\
\hline Empidonax minimus & ND & TR & - & - & $\mathrm{LC}$ & - \\
\hline Empidonax difficilis & ND & VI & - & - & $\mathrm{LC}$ & - \\
\hline Empidonax occidentalis & ND & $\mathrm{RR}$ & - & - & $\mathrm{LC}$ & - \\
\hline Sayornis nigricans & $\mathrm{C}$ & $\mathrm{RR}$ & - & - & $\mathrm{LC}$ & - \\
\hline Pyrocephalus rubinus & $\mathrm{R}$ & $\mathrm{RR}$ & - & - & $\mathrm{LC}$ & - \\
\hline Myiarchus tuberculifer & ND & $\mathrm{RR}$ & - & - & $\mathrm{LC}$ & - \\
\hline Myiarchus cinerascens & ND & VI & - & - & $\mathrm{LC}$ & - \\
\hline Myiarchus nuttingi & ND & $\mathrm{RR}$ & - & - & $\mathrm{LC}$ & - \\
\hline Myiarchus tyrannulus & ND & $\mathrm{RR}$ & - & - & $\mathrm{LC}$ & - \\
\hline Pitangus sulphuratus & $\mathrm{PC}$ & $\mathrm{RR}$ & - & - & $\mathrm{LC}$ & - \\
\hline Myiozetetes similis & $\mathrm{PC}$ & $\mathrm{RR}$ & - & - & $\mathrm{LC}$ & - \\
\hline Myiodynastes luteiventris & $\mathrm{PC}$ & VV & - & - & $\mathrm{LC}$ & - \\
\hline Tyrannus melancholicus & ND & $\mathrm{RR}$ & - & - & $\mathrm{LC}$ & - \\
\hline Tyrannus vociferans & ND & $\mathrm{RR}$ & - & - & $\mathrm{LC}$ & - \\
\hline Tyrannus crassirostris & $\mathrm{C}$ & $\mathrm{RR}$ & - & - & $\mathrm{LC}$ & - \\
\hline Tyrannus verticalis & ND & VI & - & - & $\mathrm{LC}$ & - \\
\hline \multicolumn{7}{|l|}{ Genera INCERTAE SEDIS } \\
\hline Pachyramphus aglaiae & $\mathrm{R}$ & $\mathrm{RR}$ & - & - & $\mathrm{LC}$ & - \\
\hline \multicolumn{7}{|l|}{ LANIIDAE } \\
\hline Lanius ludovicianus & $\mathrm{R}$ & $\mathrm{RR}$ & - & - & $\mathrm{LC}$ & - \\
\hline \multicolumn{7}{|l|}{ VIREONIDAE } \\
\hline Vireo solitarius & $\mathrm{R}$ & VI & - & - & $\mathrm{LC}$ & - \\
\hline Vireo huttoni & $\mathrm{PC}$ & $\mathrm{RR}$ & - & - & $\mathrm{LC}$ & - \\
\hline Vireo hypochryseus & $\mathrm{PC}$ & $* \mathrm{RR}$ & Oeste de México & - & $\mathrm{LC}$ & - \\
\hline \multicolumn{7}{|l|}{ CORVIDAE } \\
\hline Corvus corax & A & $\mathrm{RR}$ & - & - & $\mathrm{LC}$ & - \\
\hline \multicolumn{7}{|l|}{ HIRUNDINIDAE } \\
\hline Stelgidopteryx serripennis & $\mathrm{PC}$ & $\mathrm{RR}$ & - & - & $\mathrm{LC}$ & - \\
\hline \multicolumn{7}{|l|}{ AEGITHALIDAE } \\
\hline Psaltriparus minimus & $\mathrm{R}$ & $\mathrm{RR}$ & - & - & $\mathrm{LC}$ & - \\
\hline \multicolumn{7}{|l|}{ TROGLODYTIDAE } \\
\hline Campylorhynchus jocosus & $\mathrm{C}$ & $* \mathrm{RR}$ & Cuenca del Balsas & - & $\mathrm{LC}$ & - \\
\hline Catherpes mexicanus & MA & $\mathrm{RR}$ & - & - & $\mathrm{LC}$ & - \\
\hline
\end{tabular}


Apéndice 1. Continúa

\begin{tabular}{|c|c|c|c|c|c|c|}
\hline \multirow{2}{*}{ Taxón } & \multirow[b]{2}{*}{ Abundancia } & \multirow[b]{2}{*}{ Estacionalidad } & \multirow[b]{2}{*}{ Endemismo } & \multicolumn{3}{|c|}{ Categoría de riesgo } \\
\hline & & & & NOM-059 & $I U C N$ & CITES \\
\hline Thryothorus pleurostictus & $\mathrm{R}$ & $\mathrm{RR}$ & - & - & $\mathrm{LC}$ & - \\
\hline \multicolumn{7}{|l|}{ REGULIDAE } \\
\hline Regulus calendula & PC & VI & - & - & $\mathrm{LC}$ & - \\
\hline \multicolumn{7}{|l|}{ SYLVIIDAE } \\
\hline Polioptila caerulea & $\mathrm{C}$ & VI & - & - & $\mathrm{LC}$ & - \\
\hline Polioptila albiloris & $\mathrm{C}$ & $* \mathrm{RR}$ & - & - & $\mathrm{LC}$ & - \\
\hline \multicolumn{7}{|l|}{ TURDIDAE } \\
\hline Turdus grayi & $\mathrm{R}$ & RR & - & - & $\mathrm{LC}$ & - \\
\hline Turdus rufopalliatus & $\mathrm{R}$ & $\mathrm{RR}$ & Oeste de México & - & $\mathrm{LC}$ & - \\
\hline \multicolumn{7}{|l|}{ MIMIDAE } \\
\hline Mimus polyglottos & $\mathrm{R}$ & $\mathrm{RR}$ & - & - & $\mathrm{LC}$ & - \\
\hline Toxostoma curvirostre & $\mathrm{R}$ & RR & - & - & $\mathrm{LC}$ & - \\
\hline \multicolumn{7}{|l|}{ PTILOGONATIDAE } \\
\hline Ptilogonys cinereus & $\mathrm{R}$ & $\mathrm{RR}$ & Ambientes montanos & - & $\mathrm{LC}$ & - \\
\hline \multicolumn{7}{|l|}{ PARULIDAE } \\
\hline Vermivora celata & $\mathrm{R}$ & VI & - & - & $\mathrm{LC}$ & - \\
\hline Vermivora rufucapilla & $\mathrm{PC}$ & VI & - & - & $\mathrm{LC}$ & - \\
\hline Vermivora virginiae & $\mathrm{PC}$ & VI & - & - & $\mathrm{LC}$ & - \\
\hline Vermivora crissalis & $\mathrm{PC}$ & VI & - & PR & NT & - \\
\hline Dendroica coronata & $\mathrm{R}$ & VI & - & - & $\mathrm{LC}$ & - \\
\hline Dendroica nigrescens & $\mathrm{R}$ & VI & - & - & $\mathrm{LC}$ & - \\
\hline Dendroica palmarum & $\mathrm{R}$ & VI & - & - & $\mathrm{LC}$ & - \\
\hline Seiurus noveboracensis & $\mathrm{R}$ & VI & - & - & $\mathrm{LC}$ & - \\
\hline Seiurus motacilla & $\mathrm{R}$ & VI & - & - & $\mathrm{LC}$ & - \\
\hline Geothlypis trichas & $\mathrm{R}$ & VI & - & - & $\mathrm{LC}$ & - \\
\hline Wilsonia pusilla & $\mathrm{R}$ & VI & - & - & $\mathrm{LC}$ & - \\
\hline Basileuterus rufifrons & $\mathrm{C}$ & $* \mathrm{RR}$ & Oeste de México & - & $\mathrm{LC}$ & - \\
\hline Granatellus sp. & PC & $\mathrm{RR}$ & - & - & $\mathrm{LC}$ & - \\
\hline \multicolumn{7}{|l|}{ THRAUPIDAE } \\
\hline Piranga rubra & $\mathrm{R}$ & VI & - & - & $\mathrm{LC}$ & - \\
\hline Piranga ludoviciana & PC & VI & - & - & $\mathrm{LC}$ & - \\
\hline Thraupis episcopus & $\mathrm{R}$ & $\mathrm{RR}$ & - & - & $\mathrm{LC}$ & - \\
\hline Thraupis abbas & $\mathrm{R}$ & $\mathrm{RR}$ & - & - & $\mathrm{LC}$ & - \\
\hline \multicolumn{7}{|l|}{ EMBERIZIDAE } \\
\hline Pipilo albicollis & $\mathrm{C}$ & $* \mathrm{RR}$ & Cuenca del Balsas & - & $\mathrm{LC}$ & - \\
\hline Aimophila ruficauda & $\mathrm{R}$ & $\mathrm{RR}$ & - & - & $\mathrm{LC}$ & - \\
\hline Aimophila mystacalis & A & $\mathrm{RR}$ & Cuenca del Balsas & - & $\mathrm{LC}$ & - \\
\hline
\end{tabular}


Apéndice 1. Continúa

$$
\text { Taxón }
$$

Chondestes grammacus

CARDINALIDAE

Pheucticus chrysopeplus

Pheucticus melanocephalus

Cyanocompsa parellina

Passerina caerulea

Passerina cyanea

Passerina versicolor

Passerina ciris

ICTERIDAE

Molothrus aeneus

Icterus wagleri

Icterus pustulatus

Icterus galbula

FRINGILLIDAE

Euphonia hirundinacea

Carpodacus mexicanus
Abundancia

PC

$\mathrm{C}$

PC

$\mathrm{R}$

$\mathrm{R}$

C

A

PC

PC

$\mathrm{PC}$

C

$\mathrm{R}$

$\mathrm{R}$

PC
Estacionalidad

VI

* RR

RR

RR

$\mathrm{RR}$

VI

RR

VI

RR

RR

RR

VI

RR

RR
Categoría de riesgo

Endemismo

NOM-059

IUCN

CITES

LC

Oeste de México

LC

$\mathrm{LC}$

LC

LC

LC

LC

NT

LC

LC

LC

LC

$\mathrm{LC}$

LC

Apéndice 2. Valores de similitud avifaunística entre las AICAS consideradas (Arizmendi y Márquez-Valdelamar, 2000). Se indica el valor porcentual del índice de similitud de Jaccard

\begin{tabular}{|c|c|c|c|c|c|c|c|}
\hline & La Sepultura & $\begin{array}{l}\text { Centro de } \\
\text { Veracruz }\end{array}$ & $\begin{array}{c}\text { Zapotal- } \\
\text { Mactumatza }\end{array}$ & $\begin{array}{c}\text { Cicolma La } \\
\text { Mancha }\end{array}$ & $\begin{array}{c}\text { Los } \\
\text { Petenes }\end{array}$ & $\begin{array}{c}\text { Reserva } \\
\text { estatal Dzilám }\end{array}$ & $\begin{array}{l}\text { Ichka' } \\
\text { Ansijo }\end{array}$ \\
\hline La Sepultura & $*$ & 42.1053 & 28.6957 & 25.9259 & 32.2957 & 32.9502 & 32.9502 \\
\hline Centro de Veracruz & $*$ & * & 23.9669 & 37.7778 & 37.0518 & 38.189 & 38.189 \\
\hline Zapotal-Mactumatza & $*$ & * & $*$ & 26.087 & 26.8293 & 26.4706 & 26.4706 \\
\hline Cicolma La Mancha & $*$ & * & * & $*$ & 41.5584 & 42.4051 & 42.4051 \\
\hline Los Petenes & $*$ & * & * & $*$ & $*$ & 82.3944 & 82.3944 \\
\hline Reserva estatal Dzilám & $*$ & * & $*$ & * & * & * & 100 \\
\hline Ichka' Ansijo & $*$ & * & * & $*$ & $*$ & * & $*$ \\
\hline Laguna de Manialtepec & $*$ & * & $*$ & $*$ & $*$ & * & * \\
\hline Marismas Nacionales & $*$ & * & * & $*$ & * & * & * \\
\hline Chamela-Cuitzmala & $*$ & * & * & * & * & * & * \\
\hline Islas Marías & $*$ & * & * & $*$ & * & * & * \\
\hline Tancítaro & * & * & * & * & * & * & * \\
\hline Çoalcomán-Pómaro & $*$ & * & * & * & * & * & * \\
\hline Álamos-Río Mayo & $*$ & * & * & * & * & * & * \\
\hline Sierra de Huautla & $*$ & * & * & $*$ & * & * & * \\
\hline Cuenca Baja del Balsas & * & * & * & $*$ & * & * & * \\
\hline Valle de Tehuacán - & * & * & * & * & * & * & $*$ \\
\hline \multicolumn{8}{|l|}{ Cuicatlán } \\
\hline & $\begin{array}{c}\text { Laguna de } \\
\text { Manialtepec }\end{array}$ & $\begin{array}{l}\text { Marismas } \\
\text { Nacionales }\end{array}$ & $\begin{array}{l}\text { Chamela- } \\
\text { Cuitzmala }\end{array}$ & $\begin{array}{l}\text { Islas } \\
\text { Marias }\end{array}$ & Tancitaro & $\begin{array}{c}\text { Coalcomán- } \\
\text { Pómaro }\end{array}$ & $\begin{array}{l}\text { Alamos- } \\
\text { Río }\end{array}$ \\
\hline La Sepultura & 36.3248 & 31.9703 & 34.3874 & 15.5844 & 32.526 & 36.8231 & $\begin{array}{l}M a 58 \\
26.9504\end{array}$ \\
\hline Centro de Veracruz & 28.6853 & 31.0219 & 28.839 & 16.309 & 38.7097 & 38.7681 & 34.0741 \\
\hline Zapotal-Mactumatza & 32.6241 & 25.2809 & 27.6074 & 15.3846 & 18.9573 & 20.4878 & 17.0984 \\
\hline
\end{tabular}


Cicolma La Mancha

Los Petenes
Reserva estatal Dzilám

Ichka' Ansijo

Laguna de Manialtepec

Marismas Nacionales

Chamela-Cuitzmala

Islas Marías

Tancítaro

Coalcomán-Pómaro

Álamos-Río Mayo

Sierra de Huautla

Cuenca Baja del Balsas

Valle de Tehuacán -

Cuicatlán

La Sepultura

Centro de Veracruz

Zapotal-Mactumatza

Cicolma La Mancha

Los Petenes

Reserva estatal Dzilám

Ichka' Ansijo

Laguna de Manialtepec

Marismas Nacionales

Chamela-Cuitzmala

Islas Marías

Tancítaro

Coalcomán-Pómaro

Álamos-Río Mayo

Sierra de Huautla

Cuenca Baja del Balsas

Valle de Tehuacán -

Cuicatlán

$\begin{array}{cc}36.8056 & 30.8989 \\ 34.3023 & 28.3654 \\ 34.4633 & 31.1005 \\ 34.4633 & 31.1005 \\ * & 48.1928 \\ * & * \\ * & * \\ * & * \\ * & * \\ * & * \\ * & * \\ * & * \\ * & * \\ * & *\end{array}$
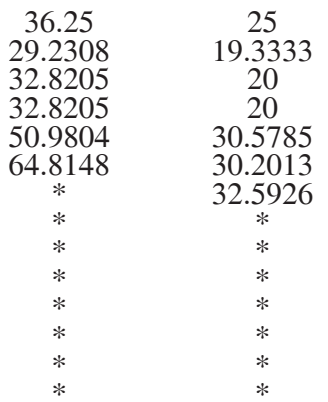

20.2765

19.4332

19.3676

20

19.3676

30.5785

33.0097

40.2715

39.8104

23.3333

32.5926

*

$*$

*

*

$\begin{array}{ccc}\begin{array}{c}\text { Sierra de } \\ \text { Huautla }\end{array} & \begin{array}{c}\text { Cuenca } \\ \text { baja del }\end{array} & \begin{array}{c}\text { Valle de } \\ \text { Tehuacán- } \\ \text { Cuicatlán }\end{array} \\ 22.2222 & 28.2258 & 24.4361 \\ 20.4 & 23.8462 & 28.3525 \\ 23.1343 & 23.1788 & 21.3415 \\ 25.8993 & 28.1046 & 26.6667 \\ 19.4286 & 22.3404 & 20.297 \\ 20 & 22.1649 & 20.7729 \\ 20 & 22.1649 & 20.7729 \\ 34.2857 & 42.1769 & 30.5882 \\ 41.7722 & 44.9704 & 35.0785 \\ 47.1831 & 49.3507 & 36.5169 \\ 32.0388 & 28.6885 & 25 \\ 36.2162 & 47.5676 & 41.5842 \\ 35.5191 & 45.4054 & 38.2353 \\ 34.3195 & 40.9091 & 41.0811 \\ * & 49.6 & 48.1481 \\ * & * & 44.4444 \\ * & * & *\end{array}$

$\begin{array}{cc}25.9804 & 22.9167 \\ 23.8298 & 20.5357 \\ 25.7384 & 20.9607 \\ 25.7384 & 20.9607 \\ 39.8964 & 30.3665 \\ 47.8261 & 41.791 \\ 53.9683 & 37.0558 \\ 24.5714 & 26.2821 \\ 67 & 47.6415 \\ * & 46.4455 \\ * & * \\ * & * \\ * & * \\ * & *\end{array}$

